\title{
Vulnerability Analysis of Road Networks Based on Hierarchical Models
}

\author{
Mei LIU ${ }^{\mathrm{a}, 1}$, Yu WANG ${ }^{\mathrm{a}}$ and Wei YAN ${ }^{\mathrm{a}}$ \\ ${ }^{a}$ China Institute of FTZ Supply Chain, Shanghai Maritime University, Shanghai, PR \\ China
}

\begin{abstract}
Road users are always expecting safe, fast, comfortable and predictable journeys both on a daily basis and on special occasions. Damage events that are predictable to a road network are challenges but those with difficulties to foresee are even greater. Vulnerability is critical in dealing with particularly high impact low chance events. In this paper the vulnerability of a road network is quantified to identify the weaknesses in the form of a road network. Theories of topology, structural vulnerability and road traffic are utilized in this paper. Based on the authors' previous work with modifications for basic concepts and variables, analysis is improved in this paper to include measures for the assessment of the form of both links and vertices. Transmittance of actual traffic flow is firstly considered for the function evaluation of a network. Traffic rerouting and reassignment is incorporated in the identification process of candidate vulnerable failure scenarios. It is proved in this paper that road networks do have weak points, single or combined, where if congested may cause great loss of function to local areas or the entire network. Findings in this paper are strong supportive points of research for the propagation of more delicate-designed and form-improved road networks in urban areas. Adaption of the current work can be made when various practical cases are involved. The vulnerability of traffic networks serving various traffic modes in a city is to be considered as future work.
\end{abstract}

Keywords. Vulnerability; road network; form; function; traffic reassignment; failure scenario.

\section{Introduction}

Threats with lower frequency occurrence that can cause parts of road networks incompetence include severe weather, natural disasters or targeted attacks. High profile events have brought enormous attention to the research on disruption to transport networks such as the airline network disrupted by the 2010 volcanic ash cloud in Iceland [1], road and railway networks submerged by the floods in Columbia in 2011[2] and Canada in 2013[3].

In[4]vulnerability is defined as the susceptibility to rare and fatal risks, whereby victims can hardly change the course of events and contribute little to recovery. The features and consequences of damage events were both addressed. In[5]it was discussed that the results of incidents should be emphasized rather than their physical nature.In many vulnerability analysis of a road network, results (consequences) of damage events

\footnotetext{
${ }^{1}$ Corresponding Author, Mail: meiliu@shmtu.edu.cn.
} 
have been evaluated by the change of 1) topological and inner characteristics of network elements and/or 2) user-oriented performances presented by the impaired network.

Topological features of a complex road networks such as vertex degree, edge betweenness and network diameter are considered to represent the excellence of the form of the networks[6-7]. Many of these measures do not reflect how well the actual flow is transmitted by the network elements whether the network is damaged or not.

The performance of a road network is usually measured by the cost paid to reach destinations by the moving flow in the network. Travel time is one of the most commonly used variables to assess the movement of traffic flow [8-9]. When another measure of the network performance, link capacity is subject to random variations due to user routing choices, it is possible for the network capacity to fluctuate around a required demand level[10]. Accessibility of a location or zone in a road network is a good measure of road network performance integrating zone attractiveness as inner features of a network such as population, number of stores and also the cost of traffic flow such as travel time, traffic volume [11-12]. In [13] the capacity bottlenecks in transport networkds are identified by capacity weighted spectral partioning analysis.

For this paper the concept of vulnerability proposed by[14]: "A road network is vulnerable if damage to a small part of the network results in the failure of a significant part or whole of it." The concept is concerned with the disproportionateness of the consequences in relation to the damage[15] and it was firstly applied to structural systems [16]. In the analysis it is the effort to cause damage events to be considered rather than probability of occurrence, predictability and nature of damage events. Consequences of damage events are measured by the change of the form and function of a road network. The form of a road network depends on its connectivity and characteristics of the network elements. The function of a road network is defined here as the service provided by network elements to vehicle traffic and local population.

\section{Topology and function of road networks}

The analysis draws upon concepts from graph theory, road traffic theory and structural vulnerability theory[17].

\subsection{Graphs of road networks}

A road network can be represented by a set of edges (i.e. a link of two directions or an arc of one direction) connected at vertices in a graph model. Defined in [21], a road network is formed by a set of road circuits. A road circuit is a closed road route that provides two non-overlapped paths between a pair of vertices. The origin and destination of a circuit are the same (i.e. traffic leaves and returns to the same origin) but without visiting any other node more than once. The environmental area, framed by a basic road circuit, is usually the urban residential area, the business district or the covering area of a city or the stacking area in a container terminal yard. A basic road circuit and its environmental area is the smallest functional unit of an operating road network.

\subsection{Forms of road networks}

As both are physical systems, a direct analogy has been used between a structure and a road network in order to examine the relationship between cause and effect[21]. The two systems are both dynamically functional ones due to connectivity among their elements. 
It is the form of either system that determines the systematic response by a changing flow to a changing potential.

Well-formedness is a common definition that describes the quality of the form of a network system. It can have various specific meanings with regard to different network systems. A well formed structure can withstand loading from any arbitrary direction. A well formed road network can provide stable and flexible services to its users such that trips can be made in any arbitrary direction i.e. from any vertex to any other within expected or accepted time. A new concept of continuance is a basis for the development of a measure of the well-formedness of a road network.

Continuance was developed through traffic modulus $(K)$ from an analogy with Young's modulus in structural mechanics[21]. Defined as traffic flow over traffic strain, traffic modulus describes the relationship between actual traffic flow and actual travel time in the system dynamic terms. Combined with the fundamental relationship between flow and travel time defined in traffic flow theory, the continuance of a road lane is defined as in Equation 1.

$$
\operatorname{tr}_{l}=\frac{Q}{\left(t_{c}-t_{f}\right) L}
$$

where $Q$ is capacity flow, the maximum sustainable flow rate at which vehicle or people reasonably can be expected to traverse a point or uniform segment of a lane or roadway during a specified time period under given conditions; $t_{c}$ is capacity travel time along the lane; $t_{f}$ is the travel time for free uninterrupted flow; $L$ is the lane length.

Continuance is always positive and directly proportional to the capacity flow of a road lane. For a road section with multiple lanes, continuance can be taken as the simple summation of each lane-though in practice there will be interference between the lanes.

\section{Form of road network elements}

\subsection{Well-formedness of a road location and a road circuit}

Vertex well-formedness depends upon the continuance, orientations of intersecting links and functional characteristics of this vertex. Functional characteristics of a vertex may include 1) its type if it is a junction (e.g. roundabout, signal-controlled etc.); 2) its size if it is a location (e.g. population, number of stores, number of activities etc.); 3) its social utility (e.g. a hospital, a school, a police station etc.). Since a global coordinate system for a road network in real world can be the north-south geographical coordinate system, the orientation of a link is reflected in a $2 \times 2$ matrix indicating the relative directionality of this link with respect to a defined co-ordinated system for the whole network. A link continuance matrix is the product of the continuance and orientation matrix of this link. The measure of vertex continuance is the determinant of the summation matrix of the continuance matrices of all the links as in Eq. 2.

$$
\begin{aligned}
{\left[M_{l i}\right] } & =\frac{Q}{\left(t_{c}-t_{f}\right) \cdot L} \cdot\left[\begin{array}{cc:cc}
\cos ^{2} \theta & \sin \theta \cos \theta & 0 & 0 \\
\sin \theta \cos \theta & \sin ^{2} \theta & 0 & 0 \\
0 & 0 & \cos ^{2} \theta & \sin \theta \cos \theta \\
0 & 0 & \sin \theta \cos \theta & \sin ^{2} \theta
\end{array}\right] \\
& =\left[\begin{array}{ll}
{[M]_{11}} & {[M]_{12}} \\
{[M]_{21}} & {[M]_{22}}
\end{array}\right]
\end{aligned}
$$

where $\theta$ is the orietation of link $l i$. 
The vertex transmittance matrix for a vertex is the summation of the link transmittance matrices for the links starting from this vertex. For a vertex with several joining links, the matrix is defined as:

$$
\left[M_{v}\right]^{i}=\left[\begin{array}{cc}
\sum_{j}\left[M_{l i}\right]^{j} & 0 \\
0 & 0
\end{array}\right] \quad j=1,2, \cdots t .
$$

where $\left[M_{a}\right]^{j}$ is the link transmittance matrix in Eq. 2 for link $j, t$ is the number of links from vertex i.

Besides vertex continuance measuring the vertex form, weights are given here to vertices to represent their relative functional importance. By referring to the functional importance of a vertex in a road network, the vertex is now considered as a zone or an area but not assumed as a relatively tiny joint point of links where it takes no time for traffic flow to pass through as in the evaluation of vertex continuance. Travel time through vertices of a roundabout or a signal-controlled junction may be different. And travel time through junctions affects flow continuance along road sections and flow continuance around a road circuit.

A reference node is defined here as a population centre such as a town or a district within a town of interest, chosen by the analyst as being of direct interest and which if disconnected from the network would cause a major loss of functionality. It is sufficient but not necessary for vertices with relatively large weights of function to be chosen as reference nodes. Similar to the ground as the reference in structural vulnerability analysis, separation from reference nodes in a road network is defined as the failure of a road network. A reference circuit is one of the independent road circuits including one or more than one reference nodes.

For the network of a city in Figure 1(a), assume that Vertex $x_{1}$ is the city centre and Vertex $x_{10}$ is a hospital and the rest vertices are residential areas with various scales of populations. Vertex $x_{1}$ and Vertex $x_{10}$ are chosen as reference nodes in the network. The function values are in boxes.

The well-formedness of a road circuit is defined as the sum of weighted vertex continuance divided by the number of vertices as in Equation 4.

$$
W f_{\text {cir }}=\frac{\sum_{i} f_{v}{ }^{i} \cdot t_{v}^{i}}{N_{v}} \quad i=\left(1,2, \cdots, N_{v}\right)
$$

where $f_{v}{ }^{i}$ is the weight of vertex $i, N_{v}$ is the total number of vertices in the circuit Cir.

In summary this proposed well-formedness measure is based on the form, number of connections and function of a vertex indicating the ease of continuation of flow through vertices with varied importance.

The damage demand for a road network is the effort required to cause the loss, by a road circuit, of its capacity to transmit traffic along a road section contained in the circuit. It is defined as being directly proportional to the traffic modulus of a road section.

\subsection{Road clusters and clustering process}

Some neighbouring circuits are clustered into a better-formed sub-network thus a road network can have a few sub-networks with different well-formedness and the relationship between them can be discovered during clustering.

The clustering process for a road network begins by identifying all of the basic road circuits and calculating their well-formedness. Clusters are grown by including the neighbouring circuits so as to result in the most increase (or least decrease) in the wellformedness. When two clusters have the same well-formedness, the other four measures are maximised during the selection of candidate circuits in the order of priority. 
A hierarchy model of a road network presents the clustering process starting from bottom to top. The higher the level the smaller is the number of clusters and the smaller amount of detailed information.

Take the network (see Figure 1(a)) with 10 vertices and 19 links for example. Vertices $x_{1}$ and $x_{10}$ are reference nodes. The functions of vertices from $x_{1}$ to $x_{10}$ are presented in boxes in Figure 1 (b). There are 10 basic circuits which are shown in Figure 2 with their well-formedness. Circuit 1, 3,6 and 8 are reference circuits. The properties of some links are given in Table 1.
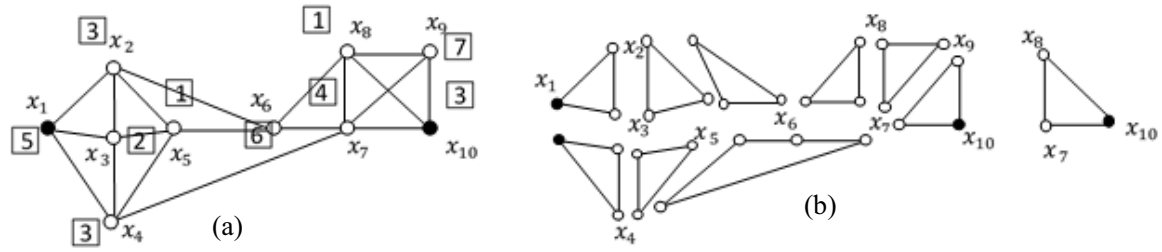

Figure 1. Basic road circuits of the road network.

Table 1. Properties of some links in the road network in Figure 1.

\begin{tabular}{c|c|c|c|c|c}
\hline \multicolumn{2}{c|}{ Link Vertices } & $\frac{Q}{\left(t_{c}-t_{f}\right)}$ & $L$ & $\theta$ & $\begin{array}{c}\text { Continuance/Damage } \\
\text { demand }\end{array}$ \\
\hline Location 1 & Location 2 & $\frac{\text { Number of vehicles } \cdot \mathrm{km}}{\mathrm{t}^{2} \cdot \text { Lane }}$ & $\mathrm{km}$ & radian & $\frac{\text { Number of vehicles }}{\mathrm{t}^{2} \cdot \text { Lane }}$ \\
\hline$x_{1}$ & $x_{2}$ & 69.04 & 1 & 0.52 & 69.04 \\
\hline$x_{1}$ & $x_{3}$ & 69.04 & 1 & 2.62 & 69.04 \\
\hline$x_{1}$ & $x_{4}$ & 69.04 & 1.73 & 2.09 & 39.86 \\
\hline$x_{9}$ & $x_{10}$ & 69.04 & 0.73 & 1.57 & 94.30 \\
\hline
\end{tabular}

The clustering process and corresponding hierarchy are illustrated in Figure 2. Two reference clusters (Cluster 16 and Cluster 17) are connected by a non-reference cluster (Cluster 15). Cluster 16 has the largest well-formedness among Cluster 15, 16 and 17. Four of the five links in the cluster, i.e. link x7- x8, x7- x10, x8- x10, x7- x9 and x9-x10 have the largest continuance (94.30) among all the links. Circuit x7-x8-x10 and x7-x9x10 have high well-formedness and they are tightly connected with a high algebraic connectivity (small number of vertices and large number of links). Vertex $\mathrm{x} 7$ in this cluster has the highest function of 7 . The other reference cluster, Cluster 17 has the lowest well-formedness among the three clusters. Five links in this cluster have lower continuance and function than those of the links in Cluster 16. The non-reference cluster, Cluster 15 has eight vertices and thirteen links that are interconnected. Redundant routes of large continuance are provided between any pair of vertices in this cluster.
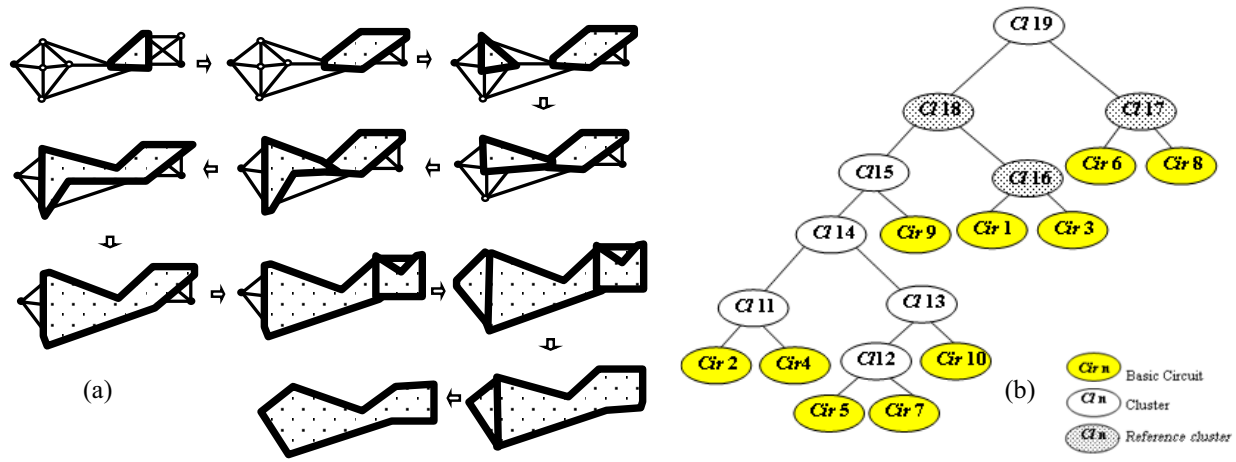

Figure 2. The clustering process (a) and the hierarchical representation(b) of the network in Figure 1. 


\section{Unzipping of road networks}

\subsection{Failure scenarios of road networks}

A failure scenario includes a series of damage events in which some vertices are disconnected from others in the circuit. Locations of damage events may result in consequences which vary from little i.e. reference nodes are still connected to each other and the well-formedness of the paths between them is not greatly damaged to extreme i.e. all of the nodes are separated from each other.

Two of the essential aspects of consequences are separateness and loss of function. Separateness is a change in the form of a road network whilst loss of function is a change in the accessibility between pairs of reference nodes. Separateness (S) is defined as a ratio of the loss in cluster well-formedness to the well-formedness of the intact network[21]. Damage to connectivity among reference nodes is reflected by loss of Function $(\mathrm{F})$ as defined in Equation 5.

$$
F=\frac{\sum_{i} f_{v}{ }^{i} \cdot f_{v}^{j}-\sum_{m} f_{v}{ }^{m} \cdot f_{v}^{n}}{\sum_{i} f_{v}{ }^{i} \cdot f_{v}^{j}}
$$

where $f_{v}{ }^{i}$ and $f_{v}{ }^{j}$ is the function of any pair of connected nodes $i$ and $j$ in the original network; $f_{v}{ }^{m}$ and $f_{v}{ }^{n}$ is the function of any pair of nodes $m$ and $n$ in the damage network. When $\boldsymbol{F}=\mathbf{1}$, all function of the network is lost; when $\boldsymbol{F}=\mathbf{0}$, the network is intact; the higher value of $\boldsymbol{F}$, the more likely for reference nodes is to be disconnected.

Neither separateness nor loss of function can fully represent the meaning of consequences to damage events. Function loss can be great when reference nodes with large function values are separated from other vertices. Therefore consequence $(\mathrm{Co})$ is defined as equal to the maximum value of $\mathrm{S}$ and $\mathrm{F}$.

Damage demand of a damage event to a link is equal to the sum of the continuance of component lanes. For more than one damage event to corresponding links it is the sum of damage demand of each link. To compare the vulnerability between different networks, the measure of relative damage demand is used.

Vulnerability index (VI) of a failure scenario is the ratio of the consequences to the relative damage demand of that scenario. This index changes with different identified scenario rather than an internal feature with a constant value. It is non-dimensional so comparisons can be made between failure scenarios in a road network and between failure scenarios in different networks.

The unzipping process searching for failure scenarios starts from the top of the hierarchy and ends in the bottom. Each cluster at each level in the hierarchy is damaged by the separation of its sub-clusters. Each separation consists of a set of links that are identified one by one referring to the unzipping criteria defined in[21]. Those links form a candidate failure scenario.

In order to identify further damage events that will lead to more partial failure scenarios, the damaged network is re-clustered and a corresponding hierarchical model is generated after a clustering process. The next event is identified using the same unzipping process but using the newly generated hierarchy. The process of re-clustering and unzipping after each event is repeated until total separation occurs or all functionality is lost. Theoretically re-clustering and unzipping can be performed many times to identify all of the candidate scenarios for a network but we assume that two sets of analyses are sufficient. The first set of candidate scenarios are identified through the 
unzipping process without re-clustering and the second set of scenarios is generated after the first link in each scenario in the first set is removed and the network is re-clustered.

Figure 3 shows the network and its hierarchical model. Vertices 1 and 16 are reference nodes. Damage demand of each link in the network is identical as 69.04 and each link contains two lanes of opposite directions. The function value for each reference node (Vertex 1 and Vertex 10) is 10 and for the rest vertices the function values are identical as 1. Well-formedness for Circuit 1 to Circuits 9 is shown in Figure 3 (a).

Different from the authors' previous work, traffic flow distribution is firstly performed here in the unzipping process together with re-clustering. At the first step traffic flow is assigned between a pair of reference nodes as origin and destination (OD) and distributed over the network. The first damage event is identified in the aforementioned process. After every damage event the traffic flow is re-distributed. If the new traffic flow on a link exceeds the link capacity, this link is considered to be damaged as the next damage event and the network is re-clustered. If no such link appears, the second damage event is identified during the re-clustering and unzipping process.

Assume that 100 vehicles per hour are flowing from the origin, Vertex 1 to the destination, Vertex 10. The flow in this network is assigned in equilibriu $\mathrm{m}$ as presented in Figure 3(b). Assume that the capacity of each link is $80 \mathrm{veh} /$ hour.
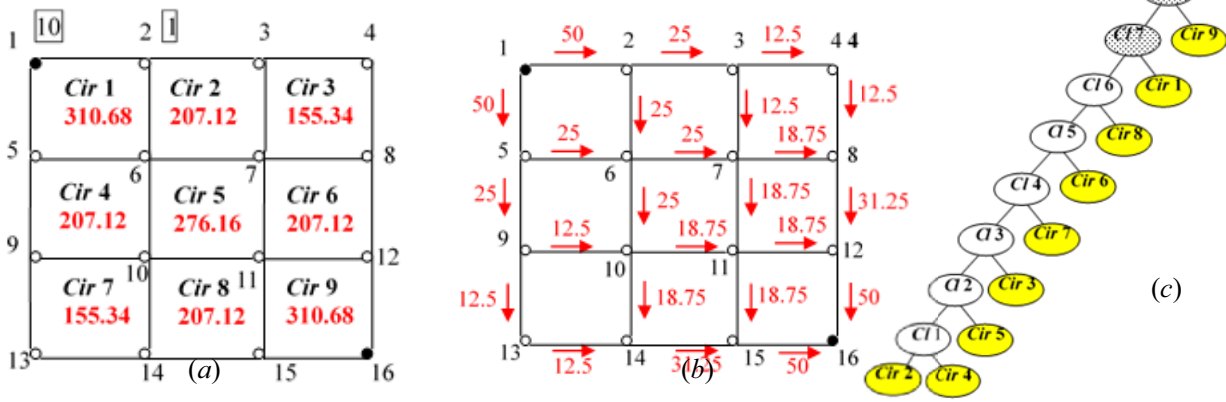

Figure 3. The grid network $(a)$, traffic flow distribution $(b)$ and the hierarchical model $(c)$.

Some candidate failure scenarios are identified in the unzipping process with and without re-clustering and are listed in Table 4.

The maximum failure scenario is Scenario a-15, i.e. the damage to link 1-2 in Table 4. The scenario is neither a total failure nor a partial failure. Since the network is symmetric horizontally, vertically and diagonally, it is apparent that there are four maximum failure scenarios, besides link 1-2, damage to link 1-5, link 12-16 and link 15-16 are the other three maximum failure scenarios respectively. Common features of the four failure scenarios are firstly, they have the smallest damage demand therefore these four failure scenarios are also minimum damage demand scenarios. Secondly the connectivity between two reference nodes and one reference node with other vertices is half damaged, i.e. one reference node is half separated from other vertices since one of the two paths connecting a reference node to the rest of the network is damaged in the failure scenarios.

Scenarios containing one of the four links (link 1-2, 1-5, 12-16 and 15-16) have large consequences, for example Scenario a-1, a-2, b-1, b-2, b-3, b-124. However relative damage demand is different for these scenarios. It can be concluded that links directly connecting reference nodes especially those with relatively high function values play 
critical roles in network vulnerability. Damage to links connecting reference nodes would either lead to great separation or large function loss of the whole network.

Table 4. Vulnerability results for the network in Figure 3.

\begin{tabular}{l|c|c|c|c}
\hline No. & $\begin{array}{c}\text { Candidate failure } \\
\text { scenario }\end{array}$ & $\begin{array}{c}\text { Relative damage } \\
\text { demand }\end{array}$ & Consequence & Vulnerability index \\
\hline
\end{tabular}

(a) without re-clustering

\begin{tabular}{l|l|l|r|r}
\hline a-1 & $12-16,15-16$ & 0.08 & 0.44 & 5.5 \\
\hline $\mathrm{a}-2$ & $1-2,1-5$ & 0.08 & 0.44 & 5.5 \\
\hline $\mathrm{a}-3$ & $5-6$ & 0.04 & 0.07 & 1.75 \\
\hline $\mathrm{a}-14$ & $8-12,11-12$ & 0.08 & 0.09 & 1.13 \\
\hline $\mathrm{a}-15$ & $1-2$ & 0.04 & 0.43 & 10.75 \\
\hline $\mathrm{a}-16$ & $2-3$ & 0.04 & 0.04 & 1 \\
\hline $\mathrm{a}-17$ & $3-4$ & 0.04 & 0.04 & 1 \\
\hline
\end{tabular}

(b) with re-clustering after damage to first link (greyed out)

\begin{tabular}{|c|c|c|c|c|}
\hline b-1 & $\mathbf{1 1}-\mathbf{1 5}, 12-16,14-15$ & 0.13 & 0.46 & 3.68 \\
\hline $\mathrm{b}-2$ & $11-15,1-2,1-5$ & 0.13 & 0.44 & 3.52 \\
\hline b-3 & $\mathbf{8}-\mathbf{1 2}, 11-12,15-16$ & 0.13 & 0.44 & 3.52 \\
\hline \multicolumn{5}{|l|}{$\cdots \cdots$} \\
\hline b-124 & $3-4,1-2$ & 0.08 & 0.46 & 5.54 \\
\hline b-125 & $3-4,9-13,13-14$ & 0.13 & 0.09 & 0.72 \\
\hline b-126 & $2-3,9-13,13-14$ & 0.13 & 0.09 & 0.72 \\
\hline
\end{tabular}

\subsection{The inclusion of traffic flow distribution}

During unzipping and re-clustering processes re-distribution of traffic flow is performed after each damage event is identified. Assume link 5-6 in Scenario a-3 in Table 4 is chosen as the first damage event. Figure 4 shows the unzipping process with the change of traffic flow on each link after the damage.

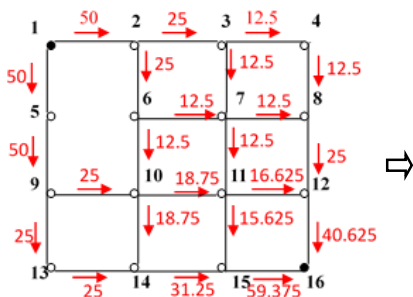

(a)

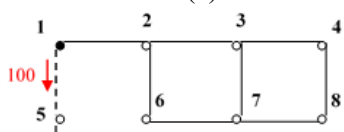

100

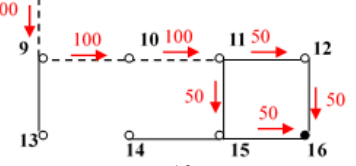

(f)

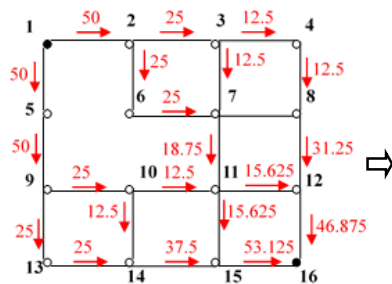

(b)

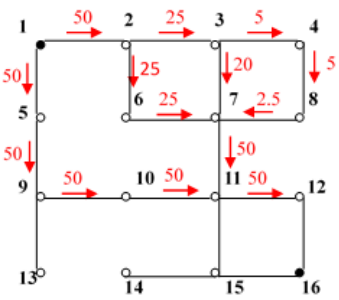

(e)

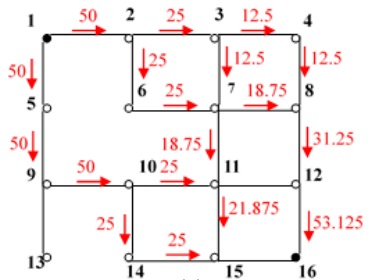

$\mathbb{1}^{(c)}$

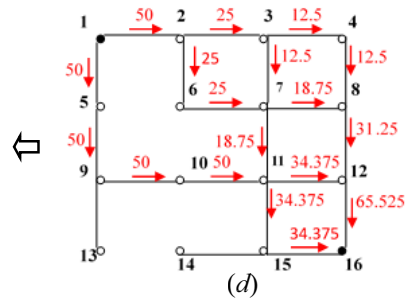

(d)

Figure 4. The unzipping process with the change of traffic flow.

From Figure 4 (a) to (e), traffic flow on links is changing and returning to a new equilibrium after each damage event. Since the link capacity is $80 \mathrm{veh} /$ hour, no link is 
identified when the traffic flow exceeds its capacity in these steps in Figure 4. The damaged links are identified in the unzipping process when re-clustering is performed after each event. In Figure 4 (f), the only one route between the reference nodes carries the whole traffic demand after link 7-11 is removed. Traffic flow on link 1-5, 5-9, 910 and 10-11 is $100 \mathrm{veh} /$ hour, which exceeds the capacity ( $80 \mathrm{veh} / \mathrm{hour}$ ) of any of the four links. The network is considered to be partially failed for the only route between the origin and destination is severely congested.

In summary by performing traffic flow re-distribution in the unzipping and reclustering processes candidate failure scenarios are generated. The vulnerability indices of those scenarios may not be high since the number of links contained in the scenarios is larger than that of scenarios identified without considering the change of actual traffic flows. However it is more similar to everyday traffic conditions when the change of traffic flow is included in the vulnerability analysis.

\section{Conclusions and future work}

1. Systems thinking, graph theory, road traffic theory and structural vulnerability theory comprise the theoretical foundations of this research. The analysis may be adjusted and applied to other transport networks such as railway networks and other network systems in trandispline engineering fields such as water pipe network, electrical networks and the Internet.

2. The vulnerability analysis for a road network is a new method to evaluate the performance of the network experiencing damage events. The analysis focuses on the form of a road network with two aspects: topology and quality. It is the consequence and effort to cause damage events rather than the nature of those events that are measured in the analysis. The analysis is adapted from the structural vulnerability theory and is firstly applied in [21]. In this paper the analysis takes functions of a road network into account.

3. In a road network, road vertices, circuits and clusters are spots and they are connected by links. For links, their well-formedness is only measured by their continuance; for spots, their well-formedness is dependent on the continuance of their joining links and the functions they display. Functional importance of road spots reflects their social roles in a road network.

4. Clustering leads to hierarchical models of networks at various levels of granularity to facilitate more efficient searching for failure scenarios. Road vertices, circuits and clusters, all as road spots are at difference levels of hierarchy. A road network hierarchy helps to abstract various levels of information about the well-formedness of parts of the network.

5. A hierarchical model of a road network is unzipped in a search process for vulnerable failure scenarios that introduces a series of damage events according to pre-defined criteria. Scenarios containing links directly connected to reference nodes are often the ones with high consequences and if damage demand to these links is small, the scenarios would have high vulnerability indices. Links directly connecting to reference nodes should be paid special attention to when considering improving the form of a road network.

6. Failure scenarios with low vulnerability indices identified in the unzipping process may cause the change of traffic flow on other links. Traffic congestion on other links, in a short time can be seen as damage events thus additional links 
should also be included in the failure scenarios. The rode network may be in partial failure under these failure scenarios.

7. The analysis results in scenarios that may help designers and engineers identify the most vulnerable parts of large road networks so that they can be redesigned, monitored or better maintained to increase robustness.

8. Results in this paper and those generated from other measures such as connectivity reliability, accessibility, vertex and link betweenness, smallworldness and limited path percolation(LPP) are supportive of each other. High vulnerable parts of a road network may have weak reliability and accessibility and weaknesses in terms of topology. Due to paper length limit, this part of research would not to be presented here.

\section{Acknowledgement}

This work is sponsored by the National Natural Science Foundation of China [grant number 71602114,71974122];Shanghai Rising-Star Program [grant number 19QA1404200], Shanghai Science \& Technology Committee Research Project [grant number 17040501700]; Shanghai Sailing Program [grant number 19YF1418800] and Shanghai Special Research Project [grant number 17DZ2280200]

\section{References}

[1] M. Guffanti, T.J. Casadevall, and K. Budding, Encounters of Aircraft with Volcanic Ash Clouds: A Compilation of Known Incidents, 1953-2009, U,S, Geological Survey, 2010.

[2] Colombia reports, Tom Heyden, 2011, Accessed: 10.02.2020. [Online]. Available: http://colombiareports,com/over-50of-colombias-road-network-affected-by-rains/

[3] National post, Canadian express, 2013, Accessed: 05.02.2020. [Online]. http://news,nationalpost,com/2013/04/22/ontario-communities-hit-by-flooding-hope-worst-now-over/

[4] G. Laurentius, The vulnerability of the city, Planning a high resilience society, In G, Weissglas (ed,)Swedish Agency for Civil Emergency and Planning (ÖCB) and Umeåuniversitet, Geographical Reports, 11, 1994.

[5] K. Berdica, An introduction to road Vulnerability: What has been done, is done and should be done, Transport Policy, Vol. 9, 2002, pp.117-127, 2002.

[6] M. Barthelemy, Spatial networks, Arxiv preprint arXiv:1010,0302, 2010.

[7] E.Lopez, R. Parchani, R. Cohen, S. Carmi and S. Havlin, Limited path percolation in complex networks, Abstract Book of the XXIII IUPAP International Conference on Statistical Physics, Genova, Italy, 2007.

[8] Q. Qiang, and A.Nagurney, A unified network performance measure with importance identification and the ranking of network components, Optimization Letters, Vol. 2, pp.127-142, 2008.

[9] E. Jenelius, Network structure and travel patterns: explaining the geographical disparities of road network vulnerability, Journal of Transport Geography, Vol. 17, pp. 234-244, 2009.

[10] A. Chen, H. Yang, K.L. Hong, and W.H. Tang, Capacity reliability of a road network: Methodology and numerical results, Transportation Research Part B, Vol. 36, pp. 225-252,2002.

[11] M.A.P. Taylor, Critical transport infrastructure in urban areas: impacts of traffic incidents assesses using accessibilitybased network vulnerability analysis, Growth and Change, Vol. 39(4), pp. 593-616, 2008.

[12] J. Sohn, Evaluating the significance of highway network links under the flood damage: an accessibility approach, Transportation Research Part A, 40, pp.491-506, 2006.

[13] M.G.H. Bell, F. Kurauchi, S. Perera, et al, Investigating transport network vulnerability by capacity weighted spectral analysis, Transportation Research Part B: Methodological, 99: 251-266, 2017.

[14] M. Liu, J. Agarwal and D. Blockley, Vulnerability of road networks, Civil Engineering and Environmental Systems, 33(2): 147-175, 2016.

[15] J. Agarwal, D.I.Blockley, N.J. Woodman, Vulnerability of systems, Civil Engineering and Environmental Systems, Vol. 18 , pp.141-165, 2001

[16] X. Wu, Vulnerability analysis of structural systems, PhD diss., Department of Civil Engineering, University of Bristol, 1991.

[17] X,Wu, D.I.Blockley, N.J. Woodman, Vulnerability of structural systems, Part I: Rings and clusters, Civil Engineering Systems, Vol. 10, pp.301-317, 1993. 